\title{
Can grain growth explain transition disks?
}

\author{
T. Birnstiel ${ }^{1,2}$, S. M. Andrews ${ }^{3}$, and B. Ercolano ${ }^{1,2}$ \\ 1 University Observatory Munich, Scheinerstr. 1, 81679 München, Germany \\ e-mail: til.birnstiel@lmu.de \\ 2 Excellence Cluster Universe, Technische Universität München, Boltzmannstr. 2, 85748 Garching, Germany \\ ${ }^{3}$ Harvard-Smithsonian Center for Astrophysics, 60 Garden Street, Cambridge, MA 02138, USA
}

Received 21 March 2012 / Accepted 21 June 2012

\begin{abstract}
Aims. Grain growth has been suggested as one possible explanation for the diminished dust optical depths in the inner regions of protoplanetary "transition" disks. In this work, we directly test this hypothesis in the context of current models of grain growth and transport.

Methods. A set of dust evolution models with different disk shapes, masses, turbulence parameters, and drift efficiencies is combined with radiative transfer calculations in order to derive theoretical spectral energy distributions (SEDs) and images.

Results. We find that grain growth and transport effects can indeed produce dips in the infrared SED, as typically found in observations of transition disks. Our models achieve the necessary reduction of mass in small dust by producing larger grains, yet not large enough to be fragmenting efficiently. However, this population of large grains is still detectable at millimeter wavelengths. Even if perfect sticking is assumed and radial drift is neglected, a large population of dust grains is left behind because the time scales on which they are swept up by the larger grains are too long. This mechanism thus fails to reproduce the large emission cavities observed in recent millimeter-wave interferometric images of accreting transition disks.
\end{abstract}

Key words. accretion, accretion disks - protoplanetary disks - stars: pre-main sequence - planets and satellites: formation circumstellar matter

\section{Introduction}

The evolution of circumstellar disks, the birthplaces of planets, is still enigmatic, even though the pioneering theoretical work on this topic was started almost 40 years ago by Lynden-Bell \& Pringle (1974). Viscous and/or gravitational stresses are the drivers of the disk accretion flows, which can be traced indirectly by the observed disk lifetimes and accretion rates (e.g., Hartmann et al. 1998; Sicilia-Aguilar et al. 2006; Hernández et al. 2007; Fedele et al. 2010).

While the general trends of declining accretion rates and disk masses can be explained by viscous evolution, a sub-set of objects called transition disks remain mysterious. These objects appear dust-depleted in their inner regions, while the outer regions resemble normal circumstellar disks (Strom et al. 1989; Skrutskie et al. 1990; Calvet et al. 2002; Espaillat et al. 2007, 2010; Andrews et al. 2011). The sizes of the dust cavities range from a few to more than 70 AU (e.g., Piétu et al. 2006; Hughes et al. 2007; Brown et al. 2008; Hughes et al. 2009; Andrews et al. 2009; Brown et al. 2009; Isella et al. 2010a; Andrews et al. 2010; Isella et al. 2010b; Andrews et al. 2011). The gas content of the cavities is still largely unknown. It may also be reduced compared to the outer disk regions as found for example by Najita et al. (2010), Dutrey et al. (2008), or Lyo et al. (2011); yet other works such as Pontoppidan et al. (2008) or Salyk et al. (2011) do detect gas inside the dust cavities.

The time scale of this transition phase of disk evolution is estimated to be of the order of a few times $10^{5}$ years (Skrutskie et al. 1990; Hartigan et al. 1990). This estimate, however, is based on the transition disk frequency, which is still only a lower limit due to the lack of spatial resolution. Classifying transition disks only based on the spectral energy distribution (SED) can be misleading because steep decreases in the dust surface density can easily be missed due to the presence of small dust particles. Some mechanisms proposed to explain observations of transition disks include planet-disk interactions (e.g., Rice et al. 2003; Zhu et al. 2011), or photo-evaporation (Clarke et al. 2001; Alexander et al. 2006; Ercolano et al. 2008). Recent imaging of disks by Andrews et al. (2011) revealed a higher fraction $(>20 \%)$ of disks with large cavities for the mm-brightest sources. Higher fractions of transition disks, indicating longer disk clearing time scales, are more difficult to explain by photoevaporation. However, the dust emission signature of photoevaporating disks has not yet been self-consistently modeled, treating grain growth physics and dust-gas feedback. It is therefore unclear whether photoevaporating disks leave a dust rich or a completely dust and gas free cavity behind (e.g., Alexander \& Armitage 2007; Garaud 2007).

The formation of large disk cavities by viscous evolution is generally problematic, even with the assumption that photoevaporation or some other mechanism is able to decouple the outer and inner disc at larger radii. Indeed the viscous time at $35 \mathrm{AU}$ for typical values for the viscosity parameter ${ }^{1}$ is $3.4 \mathrm{Myr}$. On the other hand, even at $35 \mathrm{AU}$, the time scales for grain growth and radial drift are only a couple of thousand years. Any mechanism that triggers significant changes in the dust evolution (e.g., the emergence of a pressure maximum, see Pinilla et al. 2012a) could therefore quickly induce observational signatures. The fact that grains grow and consequently become more mobile due to radial drift is likely an important part of the solution to this problem.

Recent observations by Kraus \& Ireland (2012) found a possible planetary-mass companion inside the cavity of the

\footnotetext{
1 Assuming $\alpha_{\mathrm{t}}=3 \times 10^{-3}$. Both observational and theoretical works indicate values between $10^{-3}$ and $10^{-2}$.
} 
LkCa 15 transition disk. It remains to be shown whether planets cause gaps/pressure bumps or vice versa (e.g., Kretke \& Lin 2007; Brauer et al. 2008; Pinilla et al. 2012b). Determining the origin of transition disks is therefore one of the most fundamental issues in our efforts to forge a better understanding of planet formation.

It has been suggested, although not demonstrated in any detail, that the growth and radial transport of dust could potentially explain the observed signatures of transition disks with large inner holes (e.g., Dullemond \& Dominik 2005; Tanaka et al. 2005; Najita et al. 2008; Pontoppidan et al. 2008). The observations indicate a decreased optical depth in the inner regions of transition disks both in the IR as well as in mm-observations, which does not necessarily imply diminished dust densities. Grain growth therefore offers two (related) pathways towards this end. First, particles decouple from the gas as they grow, which causes them to spiral inwards (Weidenschilling 1977; Nakagawa et al. 1986). This way, the dust optical depths are reduced by the actual removal of dust mass. Second, grain growth itself causes a decrease in the dust opacities, since larger particles emit less efficiently, which also decreses the otpical depth. Dust grains of sizes beyond a few centimeters become basically invisible to observations. However, the edges of transition disks seem to be relatively abrupt; they have been modeled as step functions (Andrews et al. 2011; Isella et al. 2012) or steep power-law profiles Isella et al. (2010b, 2012). This suggests that the environment for grain evolution must abruptly change at this point in the disk. Pressure bumps or even gaps opened by planets might be possible explanations (Lin \& Papaloizou 1986; Zhu et al. 2011). Another possibility could be the outer edges of deadzones (Gammie 1996). Dead zones are regions where the ionization fraction of the disk drops below the critical value needed to drive the magneto-rotational instability (MRI, Balbus \& Hawley 1991), the widely accepted source of turbulent viscosity.

In this work, we want to investigate whether grain growth and transport - either alone or aided by a dead zone - can be the cause of the observed transition disk signatures. In Sect. 2 we will discuss some of the equations which are crucial for the understanding of our modeling results. Section 3 describes the model setups and assumptions. The resulting simulation outcomes and simulated observations are shown and explained in Sect. 4. Our findings are discussed and summarized in Sect. 5.

\section{Background}

In the following section, we will summarize some of the results of Birnstiel et al. (2012, hereafter BKE12), which motivated the simulations presented in this paper and will help to understand the results. It was found in BKE12 that the upper end of the grain size distribution can be limited by two effects, namely fragmentation and radial drift. In the former case, due to the fact that typical impact velocities increase with grain size, the grains can only grow until they reach a size at which fragmentation sets in. The size limit for turbulent relative velocities was found to be

$a_{\mathrm{frag}} \approx 0.08 \frac{\Sigma_{\mathrm{g}}}{\rho_{\mathrm{s}} \alpha_{\mathrm{t}}} \frac{u_{\mathrm{f}}^{2}}{c_{\mathrm{s}}^{2}}$.

Here, $u_{\mathrm{f}}$ denotes the fragmentation threshold velocity and $\rho_{\mathrm{s}}$ the specific density of the dust grains. $\Sigma_{\mathrm{g}}$ is the gas surface density, $c_{\mathrm{s}}$ the sound speed and $\alpha_{\mathrm{t}}$ the turbulent viscosity parameter (Shakura \& Sunyaev 1973). The other size limit is due to radial drift: grains can only exist at a given radius if growth from smaller sizes resupplies them as fast as radial drift removes them.
In this case, the upper end of the size distribution can be approximated by (see BKE12)

$a_{\mathrm{drift}} \approx 0.35 \frac{\Sigma_{\mathrm{d}}}{\rho_{\mathrm{s}} \gamma}\left(\frac{r \Omega_{\mathrm{k}}}{c_{\mathrm{s}}}\right)^{2}$,

where $\Sigma_{\mathrm{d}}$ is the dust surface density, $\Omega_{\mathrm{k}}$ the Keplerian frequency and $\gamma$ the absolute value of the power law index of the gas pressure $P$,

$\gamma=E_{\mathrm{d}}\left|\frac{\mathrm{d} \ln P}{\mathrm{~d} \ln r}\right|$,

and we have introduced a parameter for the efficiency of radial drift, $E_{\mathrm{d}}$ where $E_{\mathrm{d}}=1$ corresponds to the fiducial literature value. In the limit $a_{\text {frag }}<a_{\text {drift }}$, fragmentation of the dust grains is the relevant size limit. In this case, small dust is constantly resupplied in the form of fragments. In the opposite case, when the radial drift barrier is the growth limiting factor, grains are removed by radial drift even before they reach sizes at which fragmentation starts to become important.

From Eqs. (1) and (2), it can be seen that the quantities which most clearly determine the time evolution of the size limits are $\Sigma_{\mathrm{g}}$ and $\Sigma_{\mathrm{d}}$, respectively, because the other quantities are not expected to change by orders of magnitude with time at a given radius. However, it should be noted that in this study, we do not track the viscous evolution of the gas surface density. This would lead to a further reduction of the maximum grain size. A fixed gas surface density is therefore in tune with having a best-case scenario to test the potential of the proposed mechanism. The fact that radial drift causes the dust surface density to decrease faster than the gas surface density, even if viscous evolution were to be included, means that the drift limit can become more important as the disk evolves.

The dependence of Eqs. (1) and (2) on $\Sigma_{\mathrm{g}}$ and $\Sigma_{\mathrm{d}}$ also causes both size limits to decrease with radius, as observed by Banzatti et al. (2011) and Guilloteau et al. (2011). If the largest grain size at a given radius is limited by radial drift, dust collision velocities are not high enough to cause fragmentation (BKE12). The drifting grains therefore sweep up the small dust without resupplying it by fragmentation. This means that drift-limited grain growth could naturally lead to conditions where the inner regions are devoid of small dust (due to lack of fragmentation), while small grains are still present in the outer disk (due to lower grain size limits in the outer regions).

The most important and uncertain parameters are $u_{\mathrm{f}}$, the collision velocity at which fragmentation sets in, and the turbulent state of the disk, which is described by the parameter $\alpha_{\mathrm{t}}$. Estimates for $u_{\mathrm{f}}$ range from a few up to $35 \mathrm{~m} \mathrm{~s}^{-1}$ (see, Wada et al. 2008; Paszun \& Dominik 2009; Blum \& Wurm 2008, and references therein). We use values of 3 and $10 \mathrm{~m} \mathrm{~s}^{-1}$, because icy aggregates are expected to fragment only at larger velocities compared to the $\sim 1 \mathrm{~m} \mathrm{~s}^{-1}$, found for silicate dust grains (Blum \& Muench 1993; Blum \& Wurm 2008).

\section{Modeling approach}

\subsection{Dust evolution}

In this work, we use a vertically averaged dust evolution code which tracks the radial and size evolution of the dust surface density of the disk. Effects of coagulation, fragmentation, and cratering as well as radial drift, gas drag, and turbulent mixing are taken into account. For any details of the dust evolution model, we refer to Birnstiel et al. (2010). 


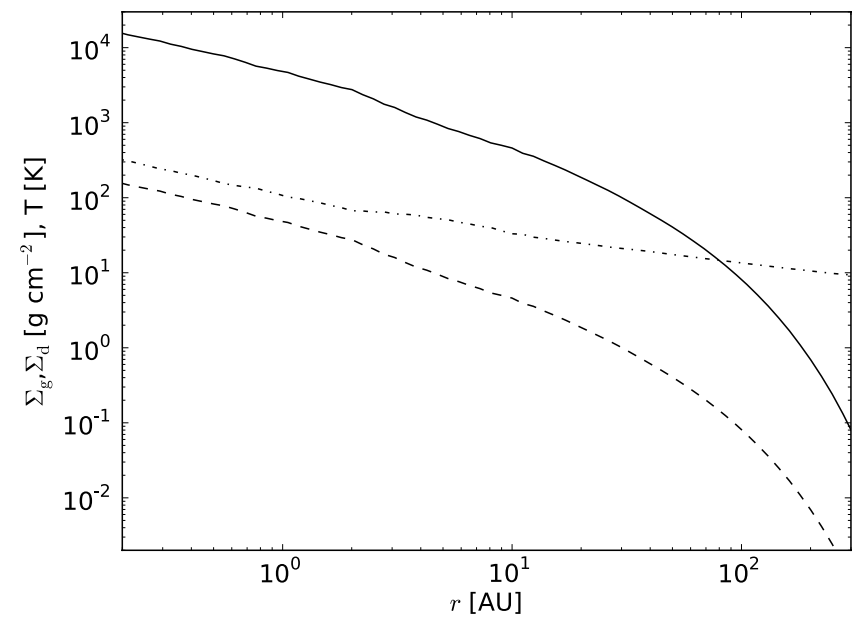

Fig. 1. Initial conditions of the simulations without a dead zone. Solid and dashed lines denote the gas and dust surface density in $\mathrm{g} \mathrm{cm}^{-2}$, respectively. The dash-dotted line denotes the mid-plane temperature.

We model both active disks, where the complete disk is MRI active as well as disks with MRI-inactive regions, the socalled dead zones. The properties and even the mere existence of dead zones is still an active matter of debate. In order to investigate whether a dead-zone can produce the observational appearance of a protoplanetary disk, we have to make some simplifications and assumptions. Therefore, we include the two most prominent effects of a dead zone which can alter the evolution of dust, namely the decreased amount of turbulence and increased gas surface density in the MRI inactive regions.

For the gas disk, we use a fixed surface density profile with a constant mass accretion rate up to a characteristic radius $r_{\mathrm{c}}$,

$\Sigma_{\mathrm{g}}(r) \propto \frac{\Omega_{\mathrm{k}}}{\alpha_{\mathrm{t}} c_{\mathrm{s}}^{2}} \cdot\left(1-\sqrt{\frac{r_{\mathrm{in}}}{r}}\right) \cdot \exp \left(-\frac{r}{r_{\mathrm{c}}}\right)$,

where $r_{\text {in }}$ is the innermost radius of the disk. In order to mimic the effects of a dead zone, we use an alpha profile of

$\alpha_{\mathrm{t}}(r)=\alpha_{\mathrm{D}}-\left(\alpha_{\mathrm{D}}-\alpha_{\mathrm{A}}\right) \cdot \begin{cases}\frac{1}{2} \exp \left(\frac{r-r_{\mathrm{dz}}}{\Delta r}\right) & \text { if } r \leq r_{\mathrm{dz}} \\ 1-\frac{1}{2} \exp \left(-\frac{r-r_{\mathrm{dz}}}{\Delta r}\right) & \text { if } r>r_{\mathrm{dz}}\end{cases}$

which represents a smooth transition at $r_{\mathrm{dz}}$ from the turbulence parameter in the dead zone $\alpha_{\mathrm{D}}$ to the active one $\alpha_{\mathrm{A}}$ over a transition width $\Delta r$, which we arbitrarily set to $1 \mathrm{AU}$. This jump in $\alpha_{\mathrm{t}}$ causes also a jump in the gas surface density profile, as can be seen from Eq. (4).

The initial condition for the dust surface density is given by a constant dust-to-gas ratio in $\mu \mathrm{m}$ sized grains. The temperature profile is derived from two-dimensional radiative transfer calculations (see Sect. 3.3) and is kept fixed throughout the simulation. As an example, the initial gas and dust surface densities and the initial temperature profile of simulation A2 is shown in Fig. 1.

\subsection{Vertical structure}

Following the results from Dubrulle et al. (1995), the grain evolution code used in this work assumes a Gaussian vertical distribution for the dust, where the scale heights depend on the grain size (see Birnstiel et al. 2010). This assumption is very accurate at the mid-plane, but it deviates in the surface layers of the disk. It can therefore be used for the dust size evolution, which mostly depends on the mid-plane values. However, to derive proper observables from the simulation outcome, the twodimensional dust density distribution needs to be reconstructed from the dust surface densities.

To this end, the vertical structure is assumed to be stationary and isothermal and we solve numerically for the equilibrium between vertical mixing and dust settling,

$\frac{\partial \rho_{\mathrm{d}}}{\partial t}=-\frac{\partial}{\partial z}\left[\rho_{\mathrm{d}} u_{\mathrm{sett}}-D_{\mathrm{d}} \rho_{\mathrm{g}} \frac{\partial}{\partial z}\left(\frac{\rho_{\mathrm{d}}}{\rho_{\mathrm{g}}}\right)\right]=0$,

where $\rho_{\mathrm{d}}$ and $\rho_{\mathrm{g}}$ are the dust and gas densities,

$D_{\mathrm{d}}=\frac{v_{\mathrm{g}}}{1+\mathrm{St}^{2}}$

is the dust diffusivity (Youdin \& Lithwick 2007); we assume that the gas diffusivity equals the gas viscosity $v_{\mathrm{g}}$. St is the particle Stokes number and

$u_{\mathrm{sett}}=\frac{3 \sqrt{\pi} m z \Omega_{\mathrm{k}}^{2}}{8 \sqrt{2} \rho_{\mathrm{g}} \pi a^{2} c_{\mathrm{s}}}$

is the settling velocity for spherical grains in the Epstein regime (Nakagawa et al. 1986). We denote the grain masses and radii as $m$ and $a$, respectively. The resulting two-dimensional density distribution of each grain size is then used in the radiative transfer calculations.

\subsection{Radiative transfer calculations}

The dust evolution models described above need to be converted into synthetic data products to assess how well they can reproduce the key observational properties of transition disks. For each model, we compute a broadband SED and high resolution millimeter-wave continuum image following the basic procedures outlined by (Andrews et al. 2009, 2011). The dust density structure $\rho_{\mathrm{d}}$ for each grain size $a$ was re-sampled onto a fixed grid in spherical coordinates, with high resolution refinements near the disk midplane and around regions of strong optical depth gradients (i.e., at the inner edge near 0.1 AU and the outer boundary of the dead zone). Absorption and scattering opacities for each grain size were calculated with a Mie code, assuming a population of segregated spheres with the optical constants and material compositions advocated by Pollack et al. (1994, see also Andrews et al. 2012). We assume that stellar irradiation is the only relevant heating source, and set the stellar photosphere to have properties that are representative of typical transition disk hosts: $T_{\text {eff }}=4300 \mathrm{~K}, R_{*}=2.5 R_{\odot}$ (implying $\left.L_{*} \approx 2 L_{\odot}\right)$, and $M_{*}=1 M_{\odot}$.

The two-dimensional Monte Carlo radiative transfer code RADMC (Dullemond \& Dominik 2004) was used to simulate the propagation of radiation through each model structure and compute an internally-consistent temperature structure. That process was iterated with the dust evolution code for each model so adjustments could be made to the vertical distribution of particles of a given size. We adopted a simple convergence criterion such that the midplane temperatures did not change more than $\sim 10 \%$ between iterations (in practice, this amounted to only 1 or 2 iterations). A ray tracing algorithm was then used on the final model structure to construct a theoretical SED and $880 \mu \mathrm{m}$ continuum image for a given disk viewing geometry and distance. For simplicity, we fixed representative values for the latter; $d=140 \mathrm{pc}$, a disk inclination $i=35^{\circ}$, and major axis position angle of $155^{\circ}$ (measured east of north). The $880 \mu \mathrm{m}$ model image is used to 
Table 1. Parameters and initial conditions of the simulations.

\begin{tabular}{lcccccc}
\hline \hline Model & $\alpha_{\mathrm{A}}$ & $\alpha_{\mathrm{D}}$ & $M_{\text {disk }}\left[M_{\odot}\right]$ & $u_{\mathrm{f}}\left[\mathrm{m} \mathrm{s}^{-1}\right]$ & $r_{\mathrm{dz}}[\mathrm{AU}]$ & $E_{\mathrm{d}}$ \\
\hline A2 & $10^{-2}$ & $10^{-2}$ & 0.20 & 3.0 & - & 1.0 \\
A3 & $10^{-3}$ & $10^{-3}$ & 0.20 & 3.0 & - & 1.0 \\
A4 & $10^{-4}$ & $10^{-4}$ & 0.20 & 3.0 & - & 1.0 \\
A5 & $10^{-5}$ & $10^{-5}$ & 0.20 & 3.0 & - & 1.0 \\
D23_M05 & $10^{-2}$ & $10^{-3}$ & 0.05 & 3.0 & 35 & 1.0 \\
D25_M05 & $10^{-2}$ & $10^{-5}$ & 0.05 & 3.0 & 35 & 1.0 \\
D35_M05 & $10^{-3}$ & $10^{-5}$ & 0.05 & 3.0 & 35 & 1.0 \\
LDE & $10^{-2}$ & $10^{-5}$ & 0.05 & 3.0 & 35 & 0.1 \\
COAG & $10^{-5}$ & $10^{-5}$ & 0.20 & - & - & 0.0 \\
\hline
\end{tabular}

Notes. Models A2 through A5 are the completely active disks, models starting with D are the disks with dead zones, LDE stands for low drift efficiency, and COAG is a model with neither radial drift nor fragmentation. Other relevant initial conditions are the stellar mass $M_{\star}=1 M_{\odot}$, stellar temperature $T_{\star}=4300 \mathrm{~K}$, and $R_{\star}=2.5 R_{\odot}$.

synthesize a simulated interferometric image like those observed by the Submillimeter Array (for examples, see Andrews et al. 2011).

\subsection{Shortcomings of the modeling approach}

A vertically integrated coagulation code combined with a vertical settling-mixing equilibrium calculation is a good approximation of the vertical dust structure, as long as the disk is not layered, as it is in the presence of a dead zone. For example, a small amount of the mid-plane dust could reach the surface layers and be fragmented due to the high degree of turbulence in the active regions. Furthermore, turbulent transport in these regions can be much stronger than in the mid-plane (Turner et al. 2010). Due to the fact that our model only uses the (possibly very low) mid-plane turbulence parameter, this model represents a "best case" scenario in favor of the removal of small grains. For simplicity, the evolution of the gas surface density and also the dependence of the MRI turbulence on the dust distribution (e.g., Sano et al. 2000) are not treated in this work.

It is important to note that other obstacles for particle growth at smaller sizes have been suggested, e.g., bouncing (Güttler et al. 2010; Zsom et al. 2010) or charging effects (Okuzumi 2009). Observations, however, do find ample evidence of the presence of both large (Testi et al. 2001; Natta et al. 2004; Rodmann et al. 2006; Ricci et al. 2010) and small grains (e.g., Watson et al. 2007, and references therein) in protoplanetary disks, which suggest that these barriers have been overcome. The question remains whether the radial drift and the fragmentation barrier are indeed the upper limits of the size distribution. At least fragmentation seems necessary in order to explain the observed presence of small dust (see Dullemond \& Dominik 2005), however the expected rate of radial drift is too high to account for the observed disk lifetimes (Weidenschilling 1977; Brauer et al. 2007). On the other hand, different sizes as well as different profiles of the dust and the gas disk (Panić et al. 2009; Andrews et al. 2012) are indicating that to some extent radial drift is at work in circumstellar disks. Possibly reduced drift rates (e.g., Johansen et al. 2006) or pressure traps (Pinilla et al. 2012a) are needed to reconcile theory and observations. To account for these open issues, we will also present models with reduced or without radial drift and without fragmentation (see Sect. 4.1).

\section{Results}

In this section we will discuss the results of two parameter studies: one of entirely MRI-active disks with varied levels of turbulence, and another which mimics MRI-inactive regions (dead zones) in which we vary the turbulence in both the MRI-active and dead regions as well as the efficiency of radial drift. A summary of the models and parameters is given in Table 1 .

\subsection{Growth and transport in smooth disk profiles}

\subsubsection{Evolution of the dust distribution}

In this first parameter study, we investigate how the possible transition from a fragmentation dominated $\left(a_{\mathrm{f}}<a_{\mathrm{d}}\right)$ to a drift dominated $\left(a_{\mathrm{d}}<a_{\mathrm{f}}\right)$ size distribution is reflected in the SED of a typical disk profile. To this end we set up different simulations with the same initial conditions and vary the constant turbulence parameter $\alpha_{\mathrm{t}}$ from $10^{-2}$ to $10^{-5}$ in simulations A2 to A5, respectively. According to Eq. (1), this variation in $\alpha_{\mathrm{t}}$ shifts the fragmentation barrier with respect to the drift barrier (Eq. (2)) by three orders of magnitude in size. Thus the maximum size of the dust grains in the simulation with the highest $\alpha_{\mathrm{t}}$ value of $10^{-2}$ (i.e., simulation A2) is set by fragmentation, while the drift limit becomes more important as we go to lower turbulence values. In this parameter study, we vary only the turbulence parameter, however it is important to note that the same or similar effects can be achieved by varying the initial dust-to-gas mass ratio $\Sigma_{\mathrm{d}} / \Sigma_{\mathrm{g}}$ (for $\Sigma_{\mathrm{d}} \ll \Sigma_{\mathrm{g}}$ ), or the fragmentation velocity $u_{\mathrm{f}}$, since these parameters determine the initial ratio of the size limits (Eqs. (1) and (2)). Changing these parameters, a drift-limited size distribution can be achieved, even for a disk with relatively strong turbulence.

Figure 2 shows $\sigma(r, a)$, the dust surface density distributions as function of grain size and radius for the active disk simulations, A2 through A5, after 5 Myr of evolution. The quantity $\sigma(r, a)$ is defined as

$\sigma(r, a)=\int_{-\infty}^{\infty} n(r, z, a) m a \mathrm{~d} z$,

where $m=4 \pi / 3 \rho_{\mathrm{s}} a^{3}$ is the particle mass, and $n(r, z, a)$ is the dust number density distribution as a function of radius $r$, height above the mid-plane $z$, and grain size $a$. Integration of $\sigma(r, a)$ over $\ln (a)$ gives the dust surface density $\Sigma_{\mathrm{d}}(r)$. The solid red line in Fig. 2 denotes the fragmentation limit (cf. Eq. (1)), the dashed red line the drift size limit (cf. Eq. (2)).

Going from simulation A2 to A5 (see Fig. 2), it is evident that the small grain population becomes increasingly depleted. The reason for this behaviour is the following: radial drift causes 


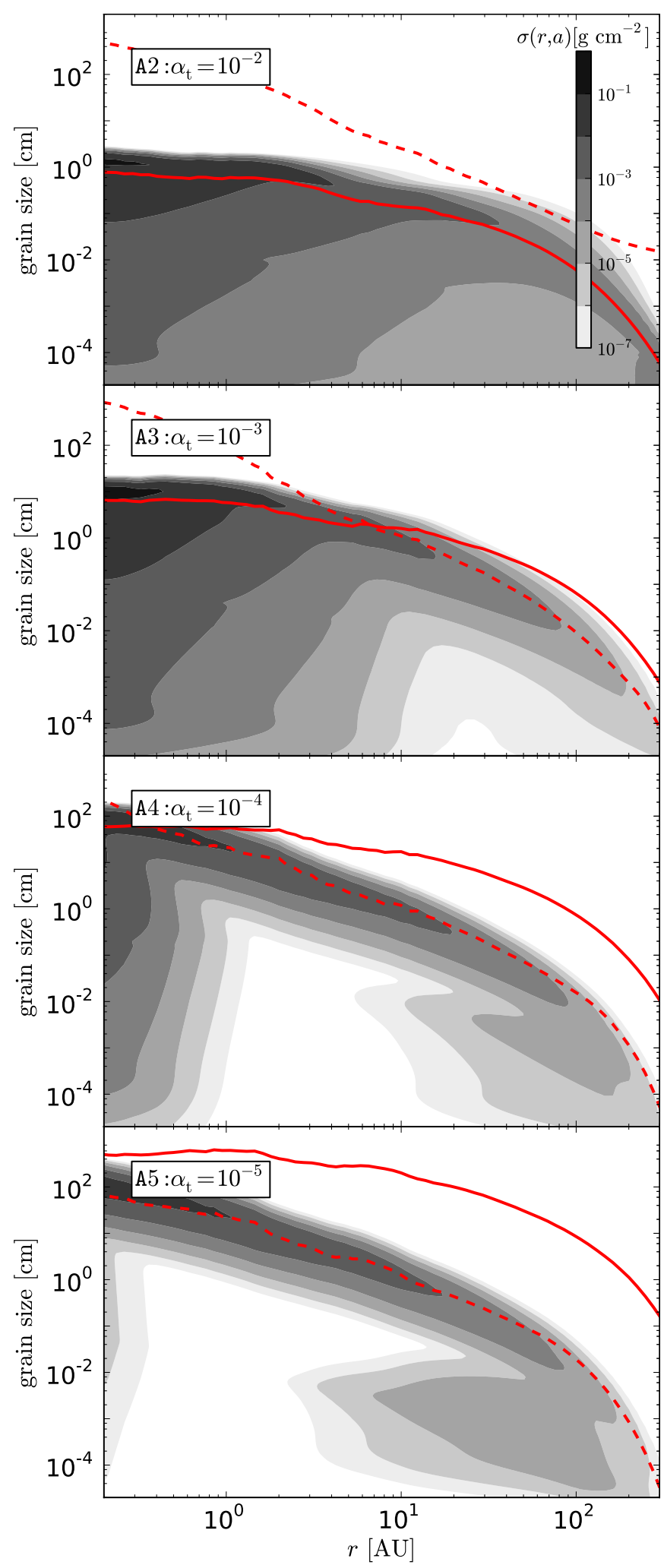

Fig. 2. Vertically integrated dust surface density distribution after $5 \mathrm{Myr}$ of evolution for the simulations without a dead zone, A2 (top) to A5 (bottom). The parameters for the simulations are shown in Table 1. The solid red line denotes the growth barrier set by grain fragmentation, the dashed red line the size limit due to radial drift.

the dust surface density to be decreased on shorter time scales than the gas surface density. The dust-to-gas ratio is therefore decreasing with time. The ratio of the two growth barriers, which in this study is initially set by the choice of $\alpha_{\mathrm{t}}$, therefore shifts with time from a fragmentation limited distribution towards a drift limited one. As described in BKE12, for a drift limited size distribution, dust collision velocities induced by radial drift decrease with the dust-to-gas ratio. Therefore, at later times, the low collision velocities prevent grain fragmentation. Consequently, small dust particles are not reproduced by fragmentation anymore, but are rather swept up by the inwarddrifting population of larger grains.

The time scale of this sweep-up can be estimated in the context of the drift-dominated two-population model of BKE12 (see Appendix A) as

$$
\begin{aligned}
& \tau_{\mathrm{D}} \simeq 600 \mathrm{yr} \frac{r}{\mathrm{AU}} \cdot \frac{2.75}{\gamma}\left(\frac{\epsilon}{10^{-2}}\right)^{-1}\left(\frac{T}{100 \mathrm{~K}}\right)^{-\frac{1}{2}} \\
& \tau_{\mathrm{T}} \simeq 1100 \mathrm{yr} \frac{r}{\mathrm{AU}} \cdot \frac{2.75}{\gamma}\left(\frac{\epsilon}{10^{-2}} \cdot \frac{T}{100 \mathrm{~K}} \cdot \frac{\alpha_{\mathrm{t}}}{10^{-3}}\right)^{-\frac{1}{2}}
\end{aligned}
$$

where $\epsilon$ is the dust-to-gas mass ratio, $\tau_{\mathrm{D}}$ and $\tau_{\mathrm{T}}$ are the sweep-up time scales for drift and turbulence induced collision velocities. The dependence on temperature $T$ and $\gamma$ is not relevant, considering that these values do not change drastically with time or radius and the dependence on $T$ is weak. The sweep-up time scale is therefore set by the quantities $r, \epsilon$, and $\alpha_{\mathrm{t}}$. The late stages of disk evolution after $5 \mathrm{Myr}$ in Fig. 2 are typically paired with dust-to-gas mass ratios of $10^{-5}$ to a few times $10^{-4}$, quite irrespective of $\alpha_{\mathrm{t}}{ }^{2}$ The radial dependence and time evolution of the dust to gas ratio is very similar to the results shown in Fig. 8 of BKE12. Therefore, the sweep-up time scales approach $\sim \mathrm{Myr}$ values beyond about $10 \mathrm{AU}$. This explains why dust at these radii is not swept up quickly. The linear dependence with $r$ means that the clearing of dust in this picture proceeds from insideout, as typically associated with other disc-clearing mechanisms (e.g., Ercolano et al. 2011). In the innermost regions, the drift size limit approaches the fragmentation limit. This causes some fragmentation of the largest particles, which is the source of the small dust population inside of $\sim 0.5 \mathrm{AU}$ for simulation $\mathrm{A} 5$ and inside of $\sim 1$ AU for simulation A4.

\subsubsection{Evolution of the SED}

The resulting SEDs and $880 \mu \mathrm{m}$ intensity profiles for all the MRI-active disk simulations are shown in Fig. 4, where the panels from left to right show the time evolution of the different models. It can be seen that the mid-IR emission of A2 and A3 are comparable up to about $20 \mu \mathrm{m}$, because even at $5 \mathrm{Myr}$ the fragmentation barrier in both cases is still at similar or even smaller grains sizes than the drift limit. Going to the lower $\alpha_{\mathrm{t}}$ values of $\mathrm{A} 4$ and $\mathrm{A} 5$ causes an ever stronger reduction of the mid-IR emission, due to the fact that the drift limit becomes significantly smaller than the fragmentation limit and the small dust grains in the hot inner regions are swept up by the drifting grains.

The outer regions show a similar effect: the emission beyond about $30 \mu \mathrm{m}$ stays high in the case of $\mathrm{A} 2$ due to the fact that the strong level of turbulence prevents the formation of larger

\footnotetext{
2 As long as the dust distribution becomes drift dominated, this leads to a self-regulating process: radial drift removes dust mass, thus lowering the dust-to-gas ratio. As the dust-to-gas ratio drops, the growth time scale and therefore also the drift time scale increases. Hence, the evolutionary time scale of the dust-to-gas ratio depends on the dust-to-gas ratio itself, and to reach a dust evolution time scale of a few Myr, the dust-to-gas ratio needs to be in the range of $10^{-5}$ to some $10^{-4}$.
} 
grains. The dust grains in this case are so small that they are well coupled to the gas. Hence, the dust is not strongly drifting, which means that more mass is retained in the outer regions and the emission therefore stays at a high level. Reducing the turbulence strength in the fragmentation limited case leads to larger particles which start to attain significant inward drift velocities. The far-IR flux is therefore decreased, because less dust mass is retained in the outer regions of the disk. This explains the strong difference between the SEDs of model A2 compared to the other models.

\subsubsection{Evolution of the (sub-)mm brightness}

The shape of the final radial intensity profiles in the 10-100 AU range can be understood by approximating $I_{v} \propto T \Sigma_{\mathrm{d}} \kappa_{v}$. For simulations A3, A4, and A5, the largest grain size between about 7 and $50 \mathrm{AU}$ is given by the drift-limit Eq. (2) and at the same time is larger than $880 \mu \mathrm{m} /(2 \pi)$. This means that the opacity $\kappa_{880}$ is roughly $\kappa_{880} \propto a_{\mathrm{dr}}^{-1} \propto T \gamma r / \Sigma_{\mathrm{d}}$. The intensity then becomes $I_{v} \propto T^{2} \gamma r$, which for a temperature profile $T \sim r^{-1 / 2}$ leads to a flat intensity profile. The entire disk of model A2 is fragmentation-dominated. This gives rise to a steeper intensity profile because the presence of large amounts of small dust means the opacity does not depend as strongly on the largest grain size. For a typical size distribution in the fragmentation case, we get $I_{v} \propto \sqrt{\Sigma_{\mathrm{g}} / T}$ which explains the slope of model A2.

\subsubsection{Conclusions for the smooth disk models}

Even though the SED signatures of the low turbulence simulations do resemble the ones of typical transition disks, there is an important drawback of this mechanism when it comes to disks with resolved holes in the (sub-)millimeter wavelength range: as pioneered by Brown et al. (2009) and confirmed by other studies such as Andrews et al. (2011), many of the transition disks (but also some of the disks without typical transition disk-like SEDs) were found to have inner dust cavities with sizes up to $70 \mathrm{AU}$. While it is easy to "hide" such cavities in the SED by relatively small amounts of small dust, the resolved optically thin (sub-)millimeter observations unambiguously reveal these features, as shown e.g., by Isella et al. (2010b) and Andrews et al. (2011).

The mechanism presented here is able to remove the small dust population; however, it does so by locking it up in a nonfragmenting population of larger dust particles. This population of grains contains still enough particles of $\sim$ centimeter sizes such that they clearly show up in the (sub-)millimeter images. So even in the best case (model A4), no cavity is seen in the simulated images (Fig. 4, bottom row). The size of the largest particles scale with the gas surface density; however, we chose a very high disk mass (20\% of the mass of the central star) in order to reach the largest possible sizes for the given disk model. Even in this case, the images shown in Fig. 4 do not resemble the observational data of e.g., Brown et al. (2009). In the following section, we will investigate how far this mechanism can be pushed by including effects which might be caused by a layered structure of the disk.

\subsection{Beyond standard dust modeling}

\subsubsection{Including effects of a layered disk structure}

So far, we have shown that the mechanism presented in this paper is able to strongly deplete the inner regions of disks in small dust grains and that a mid-IR dip in the SED, as found by observations (e.g. Skrutskie et al. 1990; Calvet et al. 2002; Espaillat et al. 2007, 2010) can be produced. In order to resemble the resolved (sub-)mm observations such as Brown et al. (2009), Andrews et al. (2011), or Lyo et al. (2011), two further features are required. First, the dust cavities also need to be observed in the (sub-)mm, i.e. for larger grains. The absence of a cavity in the simulated images of Fig. 4 indicates that the grain sizes found in our simulations are not large enough. Larger surface densities or larger fragmentation thresholds can be considered. The former option, however, is restricted by the total mass of the disk. Second, in our simulations, the dust surface density and the largest grain sizes are smooth functions of the radius, giving rise to a flat intensity profile (cf. the estimate in Sect. 4.1). This is in contrast with the observed dust cavities. These facts motivate a model which allows for higher gas surface densities and steep changes in the maximum grain size at the cavity edge.

One candidate for this behavior could be disks with dead zones, regions of low ionization which are not MRI-active. The inactive regions transport angular momentum less efficiently (Armitage 2011), which is why they can accumulate large amounts of mass, while the outer, active regions still resemble normal disks. This way, the inner, MRI-dead regions can have higher surface densities, while the transition from MRI-active to MRI-dead gives rise to strong changes in the turbulence parameter $\alpha_{\mathrm{t}}$ (e.g. Dzyurkevich et al. 2010).

In this section, we therefore take these two effects into account without any detailed modeling of the dead zone. This means that neither the vertical structure nor the viscous evolution of the dead zone are considered; only the reduced $\alpha_{\mathrm{t}}$ and the increased gas surface density $\Sigma_{\mathrm{g}}$ are treated. This is a valid approach because we are considering this as a best-case model. Other effects such as fragmentation and enhanced radial mixing in the active surface layers are working against the presented mechanism by replenishing small dust grains.

The situation where $\alpha_{\mathrm{t}}$ is higher in the outer parts of the disk and lower inside the dead zone can cause the dust distribution to be fragmentation limited outside and drift limited inside the dead zone. Models D23_MO5 and D25_MO5 simulate such a situation for $r_{\mathrm{dz}}=35 \mathrm{AU}$, but for different contrasts between the active and the dead region. The size of the dead zone is not physically motivated, but rather chosen from the typical size of the observed transition disk cavities. However, the theoretical models of Bai (2011) can account for dead zones of sizes up to $20 \mathrm{AU}$.

A comparison of the SEDs for model D23_MO5 (Fig. 5, purple SED) and A2 (Fig. 4, purple SED) highlights how the contrast of one order of magnitude in $\alpha_{\mathrm{t}}$ changes the long wavelength emission in the SED. For our choice of parameters, the outer regions start to be effected by drift, but fragmentation stays active throughout the disk. Only at $5 \mathrm{Myr}$, between around $10 \mathrm{AU}$ and the outer edge of the dead zone, the drift barrier drops slightly below the fragmentation barrier, causing a minor reduction of small grains.

We also compared models with different disk masses (not shown), but no significant differences were found for changes within a factor of a few. Going to very low disk masses of $0.005 M_{\odot}$ further reduces the maximum grain size, which is producing relatively more $\mathrm{mm}$ - and IR emission in the inner regions. Severe changes are found if the contrast in $\alpha_{\mathrm{t}}$ between the active and the dead regions is increased, because this changes the relative importance of the fragmentation and the drift barrier, as already discussed in the previous section. The simulated images in Fig. 5 (bottom row), however, do not show any signs of a cavity. The reason for this is that the peak of the size distribution 


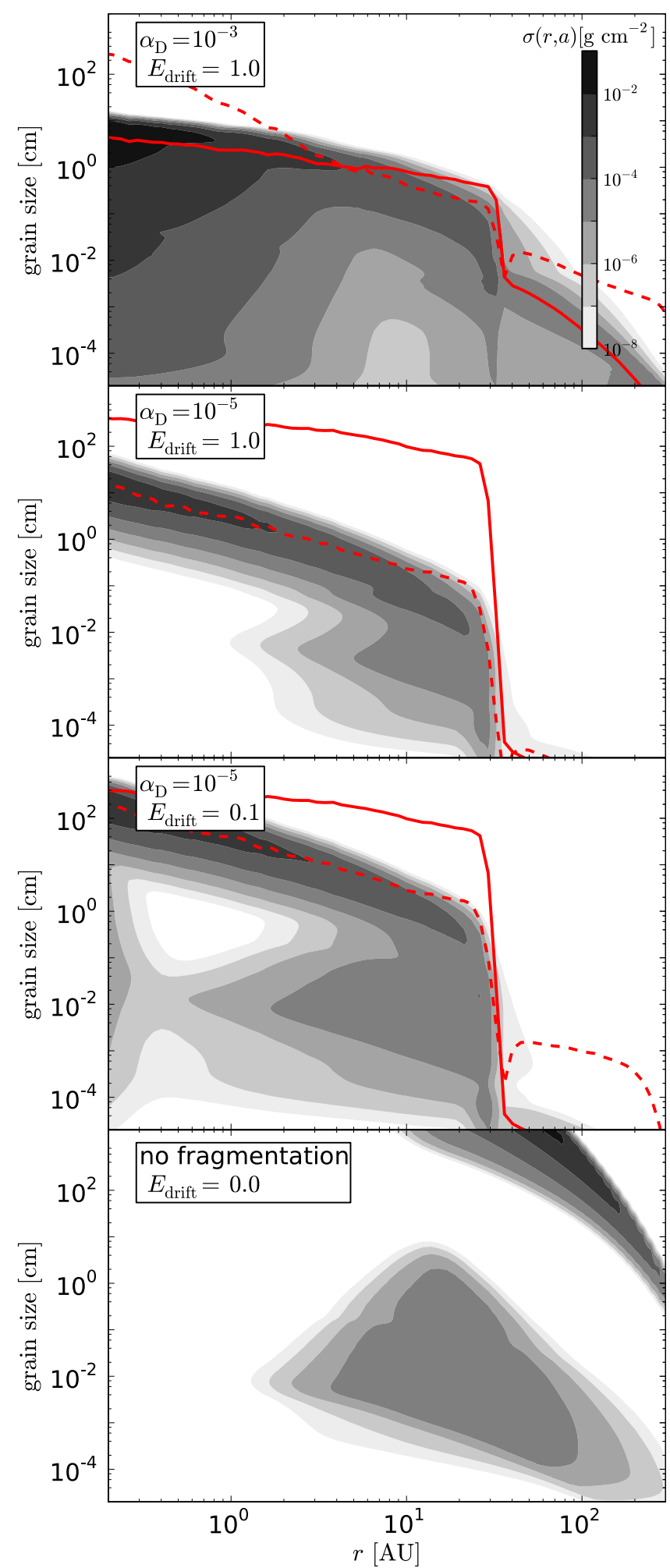

Fig. 3. Vertically integrated dust surface density distribution after $5 \mathrm{Myr}$ of evolution for simulations D23_M05 (top), D25_M05 (second panel), LDE (third panel) and COAG (bottom panel). The corresponding parameters are shown in Table 1. The solid red line denotes the growth barrier set by grain fragmentation, the dashed red line the size limit due to radial drift.

reaches sizes of around a centimeter and larger only within a few AU (see third plot from top of Fig. 3). Therefore, the surface density of dust smaller than $\sim 1 \mathrm{~cm}$ only decreases smoothly inwards of 3-8 AU, depending on the age of the disk.
In order to confirm this trend, we carried out simulations in which the size limits are pushed to even larger values. One reason for larger grains can be the efficiency of radial drift: simulations by Johansen et al. (2006) and Bai \& Stone (2010) showed that the radial drift velocity in the case of a turbulent environment can be reduced compared to the laminar, single-size results of Weidenschilling (1977) and Nakagawa et al. (1986). In order to artificially change the efficiency of radial drift, we have introduced the efficiency factor $E_{\mathrm{d}}$ in Eq. (3). From Eq. (2), it follows that $a_{\text {drift }}$ then becomes inversely proportional to the drift efficiency. This means that if radial drift is only $E_{\mathrm{d}}=0.1$ times as fast as the classical value, $a_{\mathrm{frag}}$ became $1 / E_{\mathrm{d}}=10$ times larger ${ }^{3}$.

Our simulation results with reduced drift efficiency (cf. model LDE in Table 1) show that particles do reach larger sizes in the dead zone (see third panel from top in Fig. 3). Still, the grain size is a smooth function of radius inside the dead zone. We found that going from 20 to $1 \mathrm{AU}$, the total surface density of grains smaller than one centimeter decreases only smoothly by two orders of magnitude. This means that the cavities in the mm-images cannot be explained with this mechanism. In addition to that, the reduced drift speed also increases the time scale on which small dust is swept up (cf. Eq. (11)). This results in much larger amounts of small dust being left over, as can be seen by comparing the second and third panel in Fig. 3. It also explains the less pronounced infrared dip in the SEDs (cf. Figs. 4 and 5).

The intensity shapes inside the dead zones can be understood as in the previous subsection: for all models but D23_M05, we recover a rather flat intensity profile due to the presence of large grains and a drift limited distribution. In model D23_MO5, the effective fragmentation causes a steep increase in the inner regions, as in model A3. This increase is mostly caused by changes in the opacity due to the presence of large amounts of small dust.

\subsubsection{Growth through all barriers}

It is a valid question to ask what disks would look like if our current understanding of the growth barriers and radial drift is entirely wrong. For this reason we simulated a case where grain growth is not inhibited by either fragmentation or radial drift. That is, we consider perfect sticking (without any fragmentation) and in addition, dust is only allowed to be diffused or dragged along by the gas, but not to drift (i.e., $E_{\mathrm{d}}=0$ ). Apart from those changes, all other parameters of this model are identical to A55 (see model COAG in Table 1). The resulting grain size distribution is plotted in the bottom panel of Fig. 3 and the resulting SEDs and brightness profiles are shown in Fig. 4.

In Fig. 4, it can be seen that for perfect sticking, the IR dip in the SED forms very quickly and is very pronounced, as also found by Dullemond \& Dominik (2005). While the inner parts are cleared of small dust, the growing dust particles are not able to sweep up all smaller dust further out in the disk, a population of small dust remains, as seen in the bottom panel of Fig. 3.

${ }^{3}$ It should be noted that in the fragmentation dominated limit $\left(a_{\text {frag }}<\right.$ $a_{\text {drift }}$ ), the drift velocity of the largest particles is decreased if $E_{\mathrm{d}}$ is decreased while the particle size does not change. In the drift limit $\left(a_{\text {drift }}<a_{\text {frag }}\right)$, the situation is reversed: the largest particles will grow to larger sizes until their drift time scale again equals the growth time scale. Thus the particle size is increased while the drift velocity of the largest particles is unchanged as long as their Stokes number is below unity. 

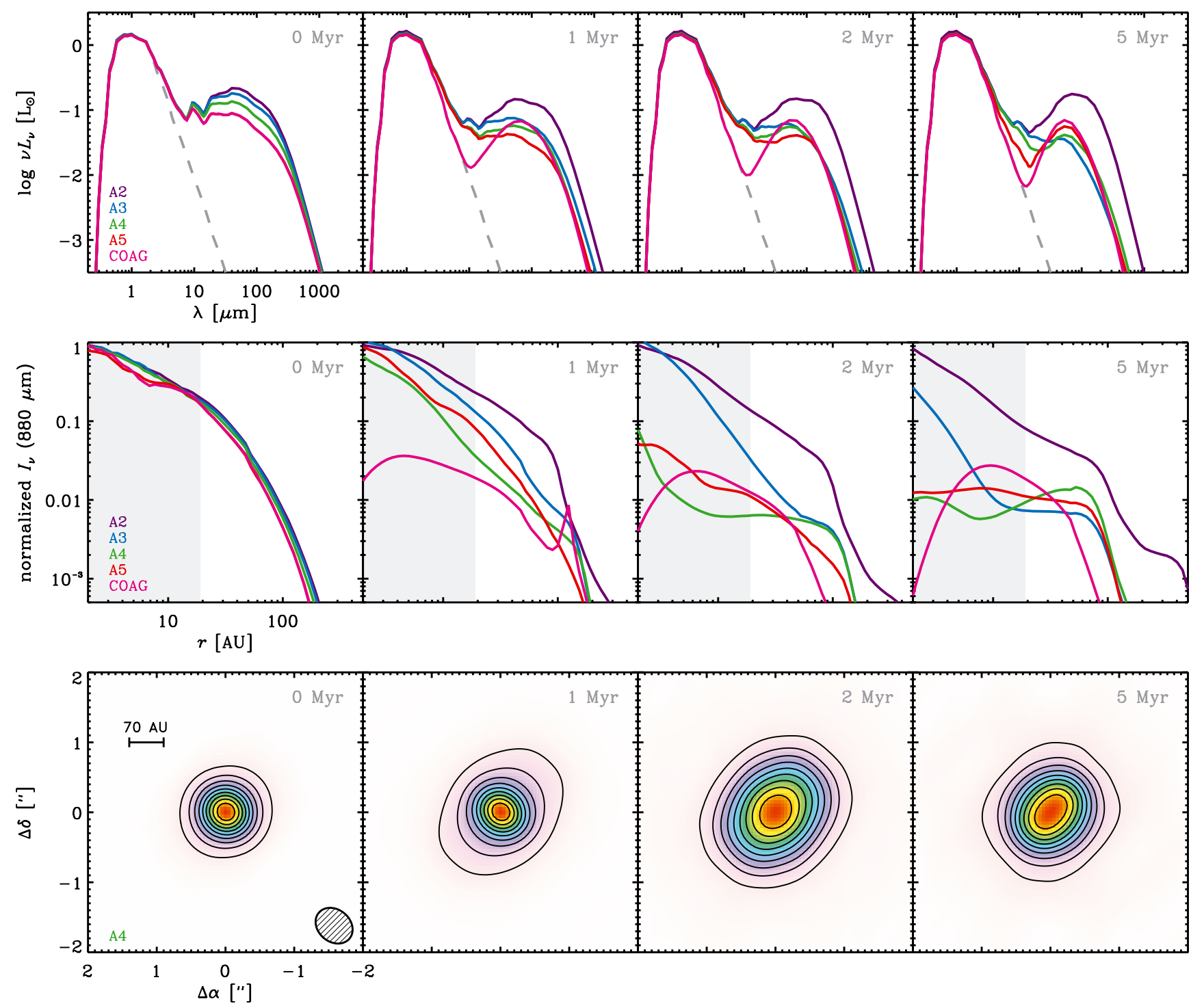

Fig. 4. Spectral energy distributions (top row), normalized radial surface brightness profiles at $880 \mu \mathrm{m}$ (middle row), and synthesized $880 \mu \mathrm{m}$ images (bottom row) corresponding to the Submillimeter Array setup of Andrews et al. (2011) for the active disk models. The line colors correspond to models A2 to A5 and model COAG, as labeled in the left panels. The dashed grey line in the SEDs represents the stellar photosphere. The columns from left to right correspond to $0,1,2$, and $5 \mathrm{Myr}$ of evolution. The shaded areas in the central row depict the resolution limits of current (pre-ALMA) interferometers. Only the results for model A4 are shown in the synthesized images, where the contours correspond to $10 \%$ levels of the peak brightness and the bar and dashed oval represent the size scale and the beam size, respectively.

We calculated the time scale for the small dust sweep-up in such a scenario (see Appendix A), which is proportional to the planetesimal size and to the quantity $r /\left(\Sigma_{\mathrm{d}} c_{\mathrm{s}}\right)$ and for our setup exceeds 1 Myr beyond 10 AU. This approximation does not include a reproduction of small dust, as would be expected for a distribution of planetesimals, which would further increase the time scale. Therefore, even a scenario with perfect sticking and no radial drift is not able to reproduce the observed large cavities in transition disks.

\section{Discussion and conclusions}

In this paper, we have investigated the ability of models of dust evolution to explain transition disks. We calculated simulated SEDs and $880 \mu \mathrm{m}$ images from the output produced by a dust evolution code for a variety of different initial conditions. For clarity, we only considered effects directly induced by growth, fragmentation and transport of dust, without pressure traps or planet induced gaps. The models were chosen to represent best-case scenarios for this mechanism to work, i.e. large gas disk masses and no viscous evolution were assumed. The only extensions to this model we have considered here are the ones which influence the grain size limits, i.e., the strength of turbulence and the gas surface density. We found that effects of grain growth can indeed produce dips in the SED such as found for many transition disks (see also Dullemond \& Dominik 2005), however they fail to reproduce the cavities in the (sub-)millimeter images such as found by Brown et al. (2009); Andrews et al. (2011); Lyo et al. (2011). Even in the extreme case where growth proceeds without any barriers and radial drift is switched off, no large inner cavities can be formed within 5 Myr of evolution.

This leads to the conclusion that disks with large inner holes cannot be caused by grain growth alone. Due to the fact that 
T. Birnstiel et al.: Can grain growth explain transition disks?
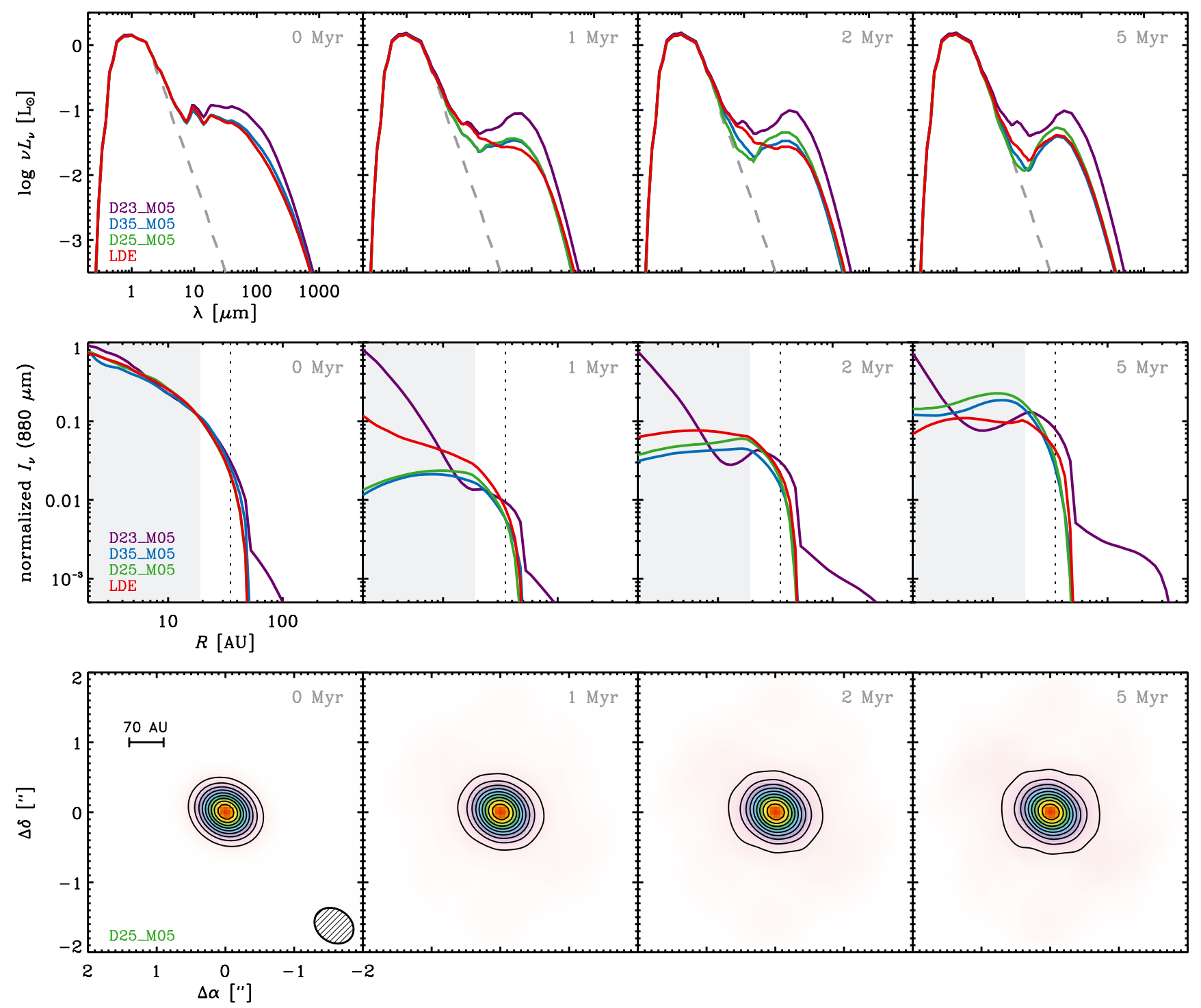

Fig. 5. Same as Fig. 4 but for the models which include dead-zones, corresponding to the simulations labeled in the left panels. The dashed vertical line in the central row marks the dead zone radius $r_{\mathrm{dz}}$. The synthesized images in the bottom row correspond to model D25_M05.

current models of disk photoevaporation (e.g. Owen et al. 2010) also fail to explain the inner cavities in the accreting objects, we propose that a combination of dust evolution with other effects such as pressure bumps, spiral arms or planet induced gaps could be the solution of the problem (see Pinilla et al. 2012b). Important questions to be answered from the observational side are: apart from the size of the cavity, is there a distinct difference between disks with small and large cavities? What is the gas content inside the cavities? And do different sizes of dust show different cavity shapes in the same object (see Dong et al. 2012)? Theoretical models will need to investigate the trapping mechanisms which effectively shepherd the dust outside the cavity, irrespective of the particle sizes.

Our findings can be summarized as follows:

- A grain size distribution for which the drift-induced grain size limit is smaller than the fragmentation induced size limit becomes inefficient in replenishing small dust due to a lack of fragmenting collisions.

- The time scale on which the remaining small dust is swept up by the largest, inward drifting grains is proportional to the distance to the central star and depends also on the dust-togas mass ratio and the turbulence strength. The dispersal of small dust is therefore from the inside out.

- Small amounts of small dust can be retained for several million years in the outer parts of the disk $(\gtrsim 10 \mathrm{AU})$ if the dustto-gas mass ratio is lower than a few times $10^{-4}$.

- Even if the inner regions are cleared of small dust via grain growth, most of the dust mass is still detectable by millimeter observations. Furthermore, the grain size is a smooth function of the stellocentric radius which means that the "observable" dust surface density decreases only slightly and the brightness profile stays approximately flat. This mechanism thus fails to reproduce the strong drop in millimeter emission which is typically observed in disks with large cavities.

- The model does not resemble the observations of large cavities, even if perfect sticking and no radial drift is assumed. The observed, sharp division between the inner and the outer regions call for a severe change in the disk properties. Pressure bumps that are strong enough to decouple both large and small dust from the accretion flow are most likely necessary to explain the observations. 
Acknowledgements. We like to thank Kees Dullemond, Chris Ormel, James Owen, and Satoshi Okuzumi for stimulating discussions. We also thank the anonymous referee for his/her very constructive and helpful criticism.

\section{Appendix A: Estimation of the sweep-up time scales}

To estimate the time scale on which small dust is swept up by larger, drifting particles, we consider the two-population model of BKE12 according to which the grain size of the drifting particles is given by

$a_{1}=\frac{2 f_{\mathrm{d}} \Sigma_{\mathrm{d}} V_{\mathrm{K}}^{2}}{\pi \rho_{\mathrm{s}} \gamma c_{\mathrm{s}}^{2}}$

where $f_{\mathrm{d}}=0.55$ and $V_{\mathrm{K}}$ is the Keplerian velocity. The drift velocity of the largest grains is then given by

$u_{1}=f_{\mathrm{d}} \epsilon V_{\mathrm{K}}$,

where $\epsilon$ is the dust-to-gas mass ratio. Assuming that the large particles contain most of the mass of the system $\left(\Sigma_{1} \simeq \Sigma_{\mathrm{d}}\right)$, and that the small particles are well mixed with the gas $\left(\mathrm{St}_{1} \lesssim \alpha_{\mathrm{t}}\right)$, we can write the change of the surface number density of small particles $N_{0}$ as (see, Birnstiel et al. 2010, Appendix A.2.)

$\dot{N}_{0}=\frac{1}{\sqrt{2 \pi} H_{\mathrm{g}}} u_{01} \sigma_{01} N_{0} N_{1}$.

Approximating the relative velocity $u_{01}$ between the two species by the drift velocity of the large grains and the collision cross section by $\sigma_{01} \simeq \pi a_{1}^{2}$, we can derive the time scale for the sweepup of small dust by drift-induced collisions

$\tau_{\mathrm{D}}=\frac{N_{0}}{\dot{N}_{0}}=\frac{8}{3} \sqrt{\frac{2}{\pi}} \frac{r}{\epsilon c_{\mathrm{s}} \gamma}$

The same calculation but using the turbulent relative velocities between the largest and smallest grains

$u_{01} \simeq \frac{3}{2} \sqrt{\frac{\alpha_{\mathrm{t}} a_{1} \rho_{\mathrm{s}} \pi}{\Sigma_{\mathrm{g}}}}$

according to Ormel \& Cuzzi (2007) yields the sweep-up time of small particles where collisions are driven by turbulence,

$\tau_{\mathrm{T}} \simeq \frac{r}{c_{\mathrm{s}}} \sqrt{\frac{f_{\mathrm{d}}}{\alpha_{\mathrm{t}} \epsilon \gamma}}$.

Comparing Eqs. (A.4) and (A.6) shows that the sweep-up of the small dust is driven by turbulent velocities, as long as

$\alpha_{\mathrm{t}} \gtrsim \frac{2}{9} f_{\mathrm{d}} \gamma \cdot \epsilon$

which is in our models approximately gives $\alpha_{\mathrm{t}} \gtrsim \frac{\epsilon}{3}$.

A similar calculation can be done for the small dust in a distribution which is dominated by planetesimals of size $a_{1}$. Using Eq. (A.3) with the cross section $\sigma_{01} \simeq \pi a_{1}^{2}$ and the relative velocities from the head wind $\Delta u_{01}=c_{\mathrm{s}}^{2} \gamma /\left(2 V_{\mathrm{K}}\right)$, we derive

$\tau_{\mathrm{P}}=\frac{8 \sqrt{2 \pi} \rho_{\mathrm{s}}}{3} \frac{a_{1} r}{\Sigma_{\mathrm{d}} c_{\mathrm{s}} \gamma}$.

For the values of our simulations (model COAG) and $a_{1}=10^{4} \mathrm{~cm}$, we find that $\tau_{\mathrm{P}} \simeq 1 \mathrm{Myr}$ at $10 \mathrm{AU}$ and increases steeply with radius. This agrees well with the fact that the planetesimals cannot efficiently sweep up the small grains beyond a few AU, as seen in the bottom panel of Fig. 3.

\section{References}

Alexander, R. D., \& Armitage, P. J. 2007, MNRAS, 375, 500

Alexander, R. D., Clarke, C., \& Pringle, J. E. 2006, MNRAS, 369, 216

Andrews, S. M., Wilner, D. J., Hughes, A. M., Qi, C., \& Dullemond, C. P. 2009, ApJ, 700, 1502

Andrews, S. M., Wilner, D. J., Hughes, A. M., Qi, C., \& Dullemond, C. P. 2010, ApJ, 723, 1241

Andrews, S. M., Wilner, D. J., Espaillat, C., et al. 2011, ApJ, 732, 42

Andrews, S. M., Wilner, D. J., Hughes, A. M., et al. 2012, ApJ, 744, 162

Armitage, P. J. 2011, ARA\&A, 49, 195

Bai, X.-N. 2011, ApJ, 739, 51

Bai, X.-N., \& Stone, J. M. 2010, ApJ, 722, 1437

Balbus, S. A., \& Hawley, J. F. 1991, ApJ, 376, 214

Banzatti, A., Testi, L., Isella, A., et al. 2011, A\&A, 525, A12

Birnstiel, T., Dullemond, C. P., \& Brauer, F. 2010, A\&A, 513, A79

Birnstiel, T., Klahr, H., \& Ercolano, B. 2012, A\&A, 539, A148

Blum, J., \& Muench, M. 1993, Icarus, 106, 151

Blum, J., \& Wurm, G. 2008, ARA\&A, 46, 21

Brauer, F., Dullemond, C. P., Johansen, A., et al. 2007, A\&A, 469, 1169

Brauer, F., Henning, T., \& Dullemond, C. P. 2008, A\&A, 487, L1

Brown, J. M., Blake, G. A., Qi, C., Dullemond, C. P., \& Wilner, D. J. 2008, ApJ, 675, L109

Brown, J. M., Blake, G. A., Qi, C., et al. 2009, ApJ, 704, 496

Calvet, N., D'Alessio, P., Hartmann, L., et al. 2002, ApJ, 568, 1008

Clarke, C., Gendrin, A., \& Sotomayor, M. 2001, MNRAS, 328, 485

Dong, R., Rafikov, R., Zhu, Z., et al. 2012, ApJ, 750, 161

Dubrulle, B., Morfill, G. E., \& Sterzik, M. F. 1995, Icarus, 114, 237

Dullemond, C. P., \& Dominik, C. 2004, A\&A, 417, 159

Dullemond, C. P., \& Dominik, C. 2005, A\&A, 434, 971

Dutrey, A., Guilloteau, S., Piétu, V., et al. 2008, A\&A, 490, L15

Dzyurkevich, N., Flock, M., Turner, N. J., Klahr, H., \& Henning, T. 2010, A\&A 515, A70

Ercolano, B., Drake, J. J., Raymond, J. C., \& Clarke, C. C. 2008, ApJ, 688, 398

Ercolano, B., Clarke, C. J., \& Hall, A. C. 2011, MNRAS, 410

Espaillat, C., Calvet, N., D'Alessio, P., et al. 2007, ApJ, 664, L111

Espaillat, C., D’Alessio, P., Hernández, J., et al. 2010, ApJ, 717, 441

Fedele, D., van den Ancker, M. E., Henning, T., Jayawardhana, R., \& Oliveira, J. M. 2010, A\&A, 510, A72

Gammie, C. F. 1996, ApJ, 457, 355

Garaud, P. 2007, ApJ, 671, 2091

Guilloteau, S., Dutrey, A., Piétu, V., \& Boehler, Y. 2011, A\&A, 529, A105

Güttler, C., Blum, J., Zsom, A., Ormel, C. W., \& Dullemond, C. P. 2010, A\&A 513, A56

Hartigan, P., Hartmann, L., Kenyon, S. J., Strom, S. E., \& Skrutskie, M. F. 1990, ApJ, 354, L25

Hartmann, L., Calvet, N., Gullbring, E., \& D’Alessio, P. 1998, ApJ, 495

Hernández, J., Hartmann, L., Megeath, T., et al. 2007, ApJ, 662

Hughes, A. M., Wilner, D. J., Calvet, N., et al. 2007, ApJ, 664, 536

Hughes, A. M., Andrews, S. M., Espaillat, C., et al. 2009, ApJ, 698, 131

Isella, A., Carpenter, J. M., \& Sargent, A. I. 2010a, ApJ, 714, 1746

Isella, A., Natta, A., Wilner, D., Carpenter, J. M., \& Testi, L. 2010b, ApJ, 725, 1735

Isella, A., Pérez, L. M., \& Carpenter, J. M. 2012, ApJ, 747, 136

Johansen, A., Klahr, H., \& Henning, T. 2006, ApJ, 636

Kraus, A. L., \& Ireland, M. J. 2012, ApJ, 745

Kretke, K. A., \& Lin, D. N. C. 2007, ApJ, 664

Lin, D. N. C., \& Papaloizou, J. 1986, ApJ, 309, 846

Lynden-Bell, D., \& Pringle, J. E. 1974, MNRAS, 168, 603

Lyo, A.-R., Ohashi, N., Qi, C., Wilner, D. J., \& Su, Y.-N. 2011, AJ, 142, 151

Najita, J. R., Crockett, N., \& Carr, J. S. 2008, ApJ, 687, 1168

Najita, J. R., Carr, J. S., Strom, S. E., et al. 2010, ApJ, 712, 274

Nakagawa, Y., Sekiya, M., \& Hayashi, C. 1986, Icarus, 67, 375

Natta, A., Testi, L., Neri, R., Shepherd, D. S., \& Wilner, D. J. 2004, A\&A, 416 179

Okuzumi, S. 2009, ApJ, 698, 1122

Ormel, C. W., \& Cuzzi, J. N. 2007, A\&A, 466, 413

Owen, J. E., Ercolano, B., Clarke, C. J., \& Alexander, R. D. 2010, MNRAS, 401, 1415

Panić, O., Hogerheijde, M. R., Wilner, D., \& Qi, C. 2009, A\&A, 501, 269

Paszun, D., \& Dominik, C. 2009, A\&A, 507, 1023

Piétu, V., Dutrey, A., Guilloteau, S., Chapillon, E., \& Pety, J. 2006, A\&A, 460, L43

Pinilla, P., Birnstiel, T., Ricci, L., et al. 2012a, A\&A, 538, A114

Pinilla, P., Benisty, M., Birnstiel, T. 2012b, A\&A, accepted

Pollack, J. B., Hollenbach, D., Beckwith, S., et al. 1994, ApJ, 421, 615

Pontoppidan, K. M., Blake, G. A., van Dishoeck, E. F., et al. 2008, ApJ, 684, 1323 
T. Birnstiel et al.: Can grain growth explain transition disks?

Ricci, L., Testi, L., Natta, A., et al. 2010, A\&A, 512, A15

Rice, W. K. M., Wood, K., Armitage, P. J., Whitney, B. A., \& Bjorkman, J. E. 2003, MNRAS, 342, 79

Rodmann, J., Henning, T., Chandler, C. J., Mundy, L. G., \& Wilner, D. J. 2006, A\&A, 446, 211

Salyk, C., Blake, G. A., Boogert, A. C. A., \& Brown, J. M. 2011, ApJ, 743, 112

Sano, T., Miyama, S. M., Umebayashi, T., \& Nakano, T. 2000, ApJ, 543, 486

Shakura, N. I., \& Sunyaev, R. A. 1973, A\&A, 24, 337

Sicilia-Aguilar, A., Hartmann, L. W., Fürész, G., et al. 2006, AJ, 132, 2135

Skrutskie, M. F., Dutkevitch, D., Strom, S. E., et al. 1990, AJ, 99, 1187

Strom, K. M., Strom, S. E., Edwards, S., Cabrit, S., \& Skrutskie, M. F. 1989, AJ, 97, 1451

Tanaka, H., Himeno, Y., \& Ida, S. 2005, ApJ, 625, 414
Testi, L., Natta, A., Shepherd, D. S., \& Wilner, D. J. 2001, ApJ, 554, 1087

Turner, N. J., Carballido, A., \& Sano, T. 2010, ApJ, 708, 188

Wada, K., Tanaka, H., Suyama, T., Kimura, H., \& Yamamoto, T. 2008, ApJ, 677, 1296

Watson, A., Stapelfeldt, K., Wood, K., \& Ménard, F. 2007, in Protostars and Planets V, eds. B. Reipurth, D. Jewitt, \& K. Keil (Tucson: University of Arizona Press), 523

Weidenschilling, S. J. 1977, MNRAS, 180, 57

Youdin, A. N., \& Lithwick, Y. 2007, Icarus, 192, 588

Zhu, Z., Nelson, R. P., Hartmann, L., Espaillat, C., \& Calvet, N. 2011, ApJ, 729, 47

Zsom, A., Ormel, C. W., Güttler, C., Blum, J., \& Dullemond, C. P. 2010, A\&A, 513, A57 